\title{
The syntactic graph of a sofic shift is invariant under shift equivalence
}

\author{
Marie-Pierre Béal* Francesca Fiorenzi* \\ Dominique Perrin*
}

\begin{abstract}
We define a new invariant for shift equivalence of sofic shifts. This invariant, that we call the syntactic graph of a sofic shift, is the directed acyclic graph of characteristic groups of the non null regular $\mathcal{D}$-classes of the syntactic semigroup of the shift.
\end{abstract}

Keywords: Automata and formal languages, symbolic dynamics.

\section{Introduction}

Sofic shifts [22] are sets of bi-infinite labels in a labeled graph. If the graph can be chosen strongly connected, the sofic shift is said to be irreducible. A particular subclass of sofic shifts is the class of shifts of finite type, defined by a finite set of forbidden blocks. Two sofic shifts $X$ and $Y$ are conjugate if there is a bijective block map from $X$ onto $Y$. It is an open question to decide whether two sofic shifts are conjugate, even in the particular case of irreducible shifts of finite type. There is a notion weaker than conjugacy, called shift equivalence (see [18, Section 7.3]). Therefore, invariants for shift equivalence are also invariants for conjugacy.

There are many invariants for conjugacy of shifts, algebraic or combinatorial, see [18, Chapter 7], [7], [17], [3]. For instance the entropy is a combinatorial invariant which gives the complexity of allowed blocks in a shift. The zeta function is another invariant which counts the number of periodic orbits in a shift.

In this paper, we define a new invariant for shift equivalence of irreducible sofic shifts. This invariant is based on the structure of the syntactic semigroup of the language of finite blocks of the shift. An irreducible sofic shift has a unique (up to isomorphisms of automata) minimal deterministic presentation, called its right Fischer cover. The syntactic semigroup $S(X)$ of an irreducible sofic shift $X$ is the transition semigroup of its right Fischer cover.

\footnotetext{
*Institut Gaspard-Monge, Université de Marne-la-Vallée, 77454 Marne-la-Vallée Cedex 2, France. \{beal,fiorenzi,perrin\}@univ-mlv.fr
} 
In general, the structure of a finite semigroup is determined by the Green's relations (denoted $\mathcal{R}, \mathcal{L}, \mathcal{H}, \mathcal{D}, \mathcal{J}$ ) [20]. Our invariant is the acyclic directed graph whose nodes the non null regular $\mathcal{D}$-classes of $S(X)$ labeled by their rank and their characteristic group. The edges correspond to the partial order $\leq_{\mathcal{J}}$ between these $\mathcal{D}$-classes. We call it the syntactic graph of the sofic shift. The result can be extended to the case of reducible sofic shifts.

We first prove the conjugacy invariance of the syntactic graph, and using this, we prove the shift equivalence invariance. The proof of the conjugacy invariance is based on Nasu's Classification Theorem for sofic shifts [19] that extends William's one for shifts of finite type. This theorem says that two irreducible sofic shifts $X, Y$ are conjugate if and only if there is a sequence of symbolic adjacency matrices of right Fischer covers $A=A_{0}, A_{1}, \ldots, A_{l-1}, A_{l}=$ $B$, such that $A_{i-1}$ and $A_{i}$ are elementary strong shift equivalent for $1 \leq i \leq l$, where $A$ and $B$ are the adjacency matrices of the right Fischer covers of $X$ and $Y$, respectively. This means that, for each $i$, there are two symbolic matrices $U_{i}$ and $V_{i}$ such that, after recoding the alphabets of $A_{i-1}$ and $A_{i}$, we have $A_{i-1}=U_{i} V_{i}$ and $A_{i}=V_{i} U_{i}$. A bipartite shift is associated in a natural way to a pair of elementary strong shift equivalent and irreducible sofic shifts [19].

The key point in our invariant is the fact that an elementary strong shift equivalence relation between adjacency matrices implies some conjugacy relations between the idempotents in the syntactic semigroup of the bipartite shift.

We show that particular classes of irreducible sofic shifts can be characterized with this syntactic invariant: the class of irreducible shifts of finite type and the class of irreducible aperiodic sofic shifts.

A related invariant characterizing reducible sofic shifts and which uses syntactic properties has been presented in [11]. It is a lattice whose vertices represent the sub-synchronizing subshifts of the shift. Some vertices of this lattice correspond to the vertices of rank 1 in our syntactic graph. Other invariants of a sofic shift, as the derived shift spaces and the depth of the shift, are given in $[21]$.

Basic definitions related to symbolic dynamics are given in Section 2.1. We refer to [18] or [14] for more details. See also [15], [16], [5] about sofic shifts. Basic definitions and properties related to finite semigroups and their structure are given Section 2.2. We refer to [20, Chapter 3] for a more comprehensive expository. Nasu's Classification Theorem is recalled in Section 2.4. We prove the conjugacy invariance of the syntactic graph in Section 3. A comparison between this syntactic invariant and some other ones which are well known, is given in Section 4. In Section 3.1, we extend the result to the case of reducible sofic shifts. In Section 5, we recall the definition of shift equivalence between sofic shifts and we prove that the syntactic graph is also invariant under shift equivalence. Part of this paper was presented at the conference STACS'04 [4]. 


\section{Definitions and background}

\subsection{Sofic shifts and their presentations}

Let $\mathcal{A}$ be a finite alphabet, i.e. a finite set of symbols. The shift map $\sigma: \mathcal{A}^{\mathbb{Z}} \rightarrow$ $\mathcal{A}^{\mathbb{Z}}$ is defined by $\sigma\left(\left(a_{i}\right)_{i \in \mathbb{Z}}\right)=\left(a_{i+1}\right)_{i \in \mathbb{Z}}$, for $\left(a_{i}\right)_{i \in \mathbb{Z}} \in \mathcal{A}^{\mathbb{Z}}$. If $\mathcal{A}^{\mathbb{Z}}$ is endowed with the product topology of the discrete topology on $\mathcal{A}$, a shift is a closed $\sigma$-invariant subset of $\mathcal{A}^{\mathbb{Z}}$.

If $X$ is a shift of $\mathcal{A}^{\mathbb{Z}}$ and $n$ a positive integer, the $n$th higher power of $X$ is the shift of $\left(\mathcal{A}^{n}\right)^{\mathbb{Z}}$ defined by $X^{n}=\left\{\left(a_{i n}, \ldots, a_{i n+n-1}\right)_{i \in \mathbb{Z}} \mid\left(a_{i}\right)_{i \in \mathbb{Z}} \in X\right\}$.

A finite automaton is a finite multigraph labeled by $\mathcal{A}$. It is denoted $\mathrm{A}=(Q, E)$, where $Q$ is a finite set of states, and $E$ a finite set of edges labeled by $\mathcal{A}$. It is equivalent to a symbolic adjacency $(Q \times Q)$-matrix $A$, where $A_{p q}$ is the finite formal sum of the labels of all the edges from $p$ to $q$. A sofic shift is the set of the labels of all the bi-infinite paths on a finite automaton. If $\mathrm{A}$ is a finite automaton, we denote by $\mathrm{X}_{\mathrm{A}}$ the sofic shift defined by the automaton A. Several automata can define the same sofic shift. They are also called presentations or covers of the sofic shift. We will assume that all presentations are essential: all states have at least one outgoing edge and one incoming edge. An automaton is deterministic if for any given state and any given symbol, there is at most one outgoing edge labeled by this given symbol. A sofic shift is irreducible if it has a presentation with a strongly connected graph. Irreducible sofic shifts have a unique (up to isomorphisms of automata) minimal deterministic presentation, that is a deterministic presentation having the fewest states among all deterministic presentations of the shift. This presentation is called the right Fischer cover of the shift.

Let $\mathrm{A}=(Q, E)$ be a finite deterministic (essential) automaton on the alphabet $\mathcal{A}$. Each finite word $w$ of $\mathcal{A}^{*}$ defines a partial function from $Q$ to $Q$. This function sends the state $p$ to the state $q$, if $w$ is the label of a path from $p$ to $q$. The semigroup generated by all these functions is called the transition semigroup of the automaton. When $\mathrm{X}_{\mathrm{A}}$ is not the full shift, the semigroup has a null element, denoted 0 , which corresponds to words which are not factors of any bi-infinite word of $\mathrm{X}_{\mathrm{A}}$. The syntactic semigroup of an irreducible sofic shift is defined as the transition semigroup of its right Fischer cover.

EXAMPle 1 The sofic shift presented by the automaton of Figure 1 is called the even shift. Its syntactic semigroup is defined by the table in the right part of the figure.

\subsection{Structure of finite semigroups}

We refer to [20] for more details about the notions defined in this section.

Given a semigroup $S$, we denote by $S^{1}$ the following monoid: if $S$ is a monoid, $S^{1}=S$. If $S$ is not a monoid, $S^{1}=S \cup\{1\}$ together with the law * defined by $x * y=x y$ if $x, y \in S$ and $1 * x=x * 1=x$ for each $x \in S^{1}$. 


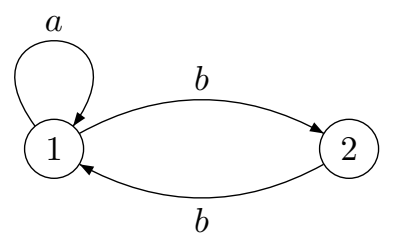

\begin{tabular}{|r|c|c|}
\hline & 1 & 2 \\
\hline$a$ & 1 & - \\
\hline$b$ & 2 & 1 \\
\hline$a b$ & 2 & - \\
\hline$b a$ & - & 1 \\
\hline$b b$ & 1 & 2 \\
\hline$b a b$ & - & 2 \\
\hline$a b a$ & - & - \\
\hline
\end{tabular}

Figure 1: The right Fischer cover of the even shift and its syntactic semigroup. Since $a a$ and $a$ define the same partial function from $Q$ to $Q$, we have $a a=a$ in the syntactic semigroup. We also have $a b a=0$ and, in general, $a b^{2 k+1} a=0$ for any nonnegative integer $k$. The word $b b$ is the identity in this semigroup.

We recall the Green's relations which are fundamental equivalence relations defined in a semigroup $S$. The four equivalence relations $\mathcal{R}, \mathcal{L}, \mathcal{H}, \mathcal{J}$ are defined as follows. Let $x, y \in S$,

$$
\begin{aligned}
x \mathcal{R} y & \Leftrightarrow x S^{1}=y S^{1}, \\
x \mathcal{L} y & \Leftrightarrow S^{1} x=S^{1} y, \\
x \mathcal{J} y & \Leftrightarrow S^{1} x S^{1}=S^{1} y S^{1}, \\
x \mathcal{H} y & \Leftrightarrow \quad x \mathcal{R} y \text { and } x \mathcal{L} y .
\end{aligned}
$$

Another relation $\mathcal{D}$ is defined by:

$$
x \mathcal{D} y \Leftrightarrow \exists z \in S \quad x \mathcal{R} z \text { and } z \mathcal{L} y .
$$

In a finite semigroup $\mathcal{J}=\mathcal{D}$. We recall the definition of the quasi-order $\leq \mathcal{J}$ :

$$
x \leq \mathcal{J} y \quad \Leftrightarrow \quad S^{1} x S^{1} \subseteq S^{1} y S^{1} .
$$

An $\mathcal{R}$-class is an equivalence class for a relation $\mathcal{R}$ (similar notations hold for the other Green's relations). An idempotent is an element $e \in S$ such that $e e=e$. A regular class is a class containing an idempotent. In a regular $\mathcal{D}$-class, any $\mathcal{H}$-class containing an idempotent is a maximal subgroup of the semigroup. Moreover, two regular $\mathcal{H}$-classes contained in a same $\mathcal{D}$-class are isomorphic (as groups), see for instance [20, Chapter 3 Proposition 1.8]. This group is called the characteristic group of the regular $\mathcal{D}$-class. The quasi-order $\leq_{\mathcal{J}}$ induces a partial order between the $\mathcal{D}$-classes (still denoted $\leq \mathcal{J}$ ). The structure of the transition semigroup $S$ is often described by the so called "egg-box" pictures of the $\mathcal{D}$-classes.

We say that two elements $x, y \in S$ are conjugate if there are elements $u, v \in$ $S^{1}$ such that $x=u v$ and $y=v u$. Two idempotents belong to a same regular $\mathcal{D}$ class if and only if they are conjugate, see for instance [20, Chapter 3 Proposition 1.12 . 
Let $S$ be a transition semigroup of an automaton $\mathrm{A}=(Q, E)$ and $x \in S$. The rank of $x$ is the cardinal of the image of $x$ as a partial function from $Q$ to $Q$. The kernel of $x$ is the partition induced by the equivalence relation $\sim$ over the domain of $x$ where $p \sim q$ if and only $p, q$ have the same image by $x$. The kernel of $x$ is thus a partition of the domain of $x$. In Figure 2, we describe the egg-box pictures for the even shift of Example 1.

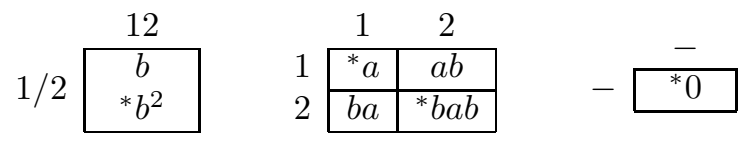

Figure 2: The syntactic semigroup of the even shift is composed of three $\mathcal{D}$-classes $D_{1}, D_{2}, D_{3}$, of rank 2, 1 and 0 , respectively, represented by the above tables from left to right. Each square in a table represents an $\mathcal{H}$-class. Each row represents an $\mathcal{R}$-class and each column an $\mathcal{L}$-class. The common kernel of the elements in each row is written on the left of the row. The common image of the elements in each column is written above the column. Idempotents are marked with the symbol $*$. Each $\mathcal{D}$-class of this semigroup is regular. The characteristic groups of $D_{1}, D_{2}, D_{3}$ are $\mathbb{Z} / 2 \mathbb{Z}$, the trivial group $\mathbb{Z} / \mathbb{Z}$ and $\mathbb{Z} / \mathbb{Z}$, respectively.

\subsection{The syntactic graph of a sofic shift}

Let $X$ be an irreducible sofic shift and $S(X)$ its syntactic semigroup. It is known that $S(X)$ has a unique $\mathcal{D}$-class of rank 1 which is regular (see [5] or [6], see also [11]).

We define a finite directed acyclic graph associated with $X$ as follows. The set of vertices of this graph is the set of non null regular $\mathcal{D}$-classes of $S(X)$, but the regular $\mathcal{D}$-class of null rank, if there is one. Each vertex is labeled by the rank of the $\mathcal{D}$-class and its characteristic group. There is an edge from the vertex associated with a $\mathcal{D}$-class $D$ to the vertex associated with a $\mathcal{D}$-class $D^{\prime}$ if and only if $D^{\prime} \leq \mathcal{J} D$. We call this acyclic graph the syntactic graph of $X$ (see Figure 3 for an example). Note that the regular $\mathcal{D}$-class of null rank, if there is one, is not taken into account in a syntactic graph. This is linked to the fact that a full shift (i.e. the set of all bi-infinite words on a finite alphabet) can be conjugate to a non full shift.

\subsection{Nasu's Classification Theorem for sofic shifts}

In this section, we recall Nasu's Classification Theorem for sofic shifts [19] (see also [18, Theorem 7.2.12]), which extends William's Classification Theorem for shifts of finite type (see [18, Theorem 7.2.7]).

Let $X \subseteq \mathcal{A}^{\mathbb{Z}}, Y \subseteq \mathcal{B}^{\mathbb{Z}}$ be two shifts and $m, a$ be nonnegative integers. A map $\phi: X \rightarrow Y$ is a $(m, a)$-block map (or $(m, a)$-factor map) if there is a map $\delta: \mathcal{A}^{m+a+1} \rightarrow \mathcal{B}$ such that $\phi\left(\left(a_{i}\right)_{i \in \mathbb{Z}}\right)=\left(b_{i}\right)_{i \in \mathbb{Z}}$ where $\delta\left(a_{i-m} \ldots a_{i-1} a_{i}\right.$ $\left.a_{i+1} \ldots a_{i+a}\right)=b_{i}$. A block map is a $(m, a)$-block map for some nonnegative integers $m, a$ (respectively called its memory and anticipation). The well known 


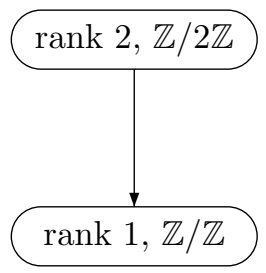

Figure 3: The syntactic graph of the even shift $X$ of Example 1. We have $D_{2} \leq_{\mathcal{J}} D_{1}$ since, for instance, $S(X)^{1} a b S(X)^{1} \subseteq S(X)^{1} b S(X)^{1}$.

theorem of Curtis, Hedlund and Lyndon [10] asserts that continuous maps commuting with the shift map $\sigma$, are exactly block maps. A conjugacy is a one-toone and onto block map (then, being a shift compact, also its inverse is a block map).

We now define the notion of strong shift equivalence between two symbolic adjacency matrices. A symbolic monomial is a formal product of several noncommuting variables. In particular, the entries of a symbolic adjacency matrix are integral combinations of symbolic monomials. In this category of matrices, we write $A \leftrightarrow B$ if $A=B$ modulo a bijection of their underlying symbolic monomials. For example we can write

$$
\left[\begin{array}{cc}
0 & b \\
b+c & 2 a
\end{array}\right] \leftrightarrow\left[\begin{array}{cc}
0 & a \\
a+d & 2 e
\end{array}\right] \leftrightarrow\left[\begin{array}{cc}
0 & b b \\
b b+c c & 2 c b
\end{array}\right]
$$

Two symbolic matrices $A$ and $B$ with entries in $\mathcal{A}$ and $\mathcal{B}$ respectively, are elementary strong shift equivalent if there is a pair symbolic matrices $(U, V)$ with entries in disjoint alphabets $\mathcal{U}$ and $\mathcal{V}$ respectively, such that $A \leftrightarrow U V$ and $B \leftrightarrow V U$.

Another equivalent formulation of this definition is the following. Let $\mathcal{A}$ and $\mathcal{B}$ be two finite alphabets. We denote by $\mathcal{A B}$ the set of words $a b$ with $a \in \mathcal{A}$ and $b \in \mathcal{B}$. Let $f$ be a map from $\mathcal{A}$ to $\mathcal{B}$. The map $f$ is extended to a morphism from finite formal sums of elements of $\mathcal{A}$ to finite formal sums of elements of $\mathcal{B}$. We say that $f$ transforms a symbolic $(Q \times Q)$-matrix $A$ into a symbolic $(Q \times Q)$ matrix $B$ if $B_{p q}=f\left(A_{p q}\right)$ for each $p, q \in Q$. Two symbolic matrices $A$ and $B$ with entries in $\mathcal{A}$ and $\mathcal{B}$ respectively, are elementary strong shift equivalent if there is a pair of symbolic matrices $(U, V)$ with entries in disjoint alphabets $\mathcal{U}$ and $\mathcal{V}$ respectively, such that there is a one-to-one map from $\mathcal{A}$ to $\mathcal{U V}$ which transforms $A$ into $U V$, and there is a one-to-one map from $\mathcal{B}$ to $\mathcal{V U}$ which transforms $B$ into $V U$.

Two symbolic adjacency matrices $A$ and $B$ are strong shift equivalent within right Fischer covers if there is a sequence of symbolic adjacency matrices of right Fischer covers

$$
A=A_{0}, A_{1}, \ldots, A_{l-1}, A_{l}=B
$$


such that for $1 \leq i \leq l$ the matrices $A_{i-1}$ and $A_{i}$ are elementary strong shift equivalent.

Theorem 2 (NAsu) Let $X$ and $Y$ be irreducible sofic shifts and let $A$ and $B$ be the symbolic adjacency matrices of the right Fischer covers of $X$ and $Y$, respectively. Then $X$ and $Y$ are conjugate if and only if $A$ and $B$ are strong shift equivalent within right Fischer covers.

EXAMPle 3 Let us consider the two (conjugate) irreducible sofic shifts $X$ and $Y$ defined by the right Fischer covers in Figure 4.
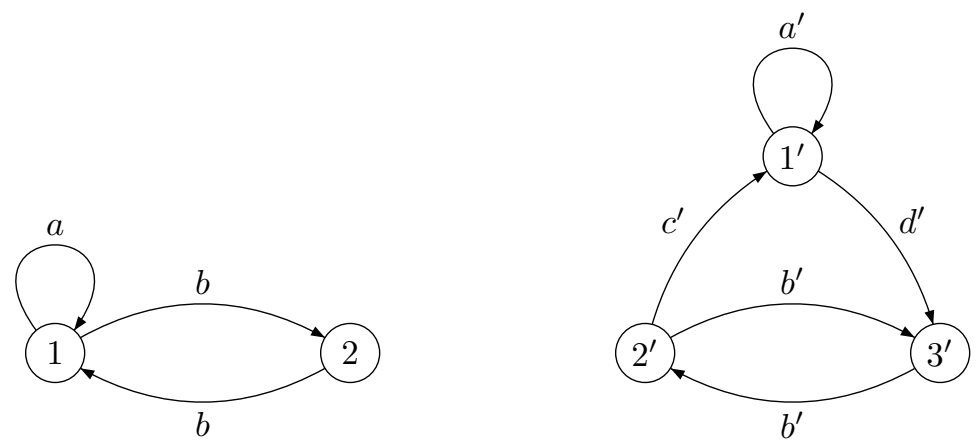

Figure 4: Two conjugate shifts $X$ and $Y$.

The symbolic adjacency matrices of these automata are respectively

$$
A=\left[\begin{array}{ll}
a & b \\
b & 0
\end{array}\right], \quad B=\left[\begin{array}{ccc}
a^{\prime} & 0 & d^{\prime} \\
c^{\prime} & 0 & b^{\prime} \\
0 & b^{\prime} & 0
\end{array}\right]
$$

Then $A$ and $B$ are elementary strong shift equivalent with

$$
U=\left[\begin{array}{ccc}
u_{1} & 0 & u_{2} \\
0 & u_{2} & 0
\end{array}\right], \quad V=\left[\begin{array}{cc}
v_{1} & 0 \\
v_{2} & 0 \\
0 & v_{2}
\end{array}\right]
$$

Indeed,

$$
U V=\left[\begin{array}{cc}
u_{1} v_{1} & u_{2} v_{2} \\
u_{2} v_{2} & 0
\end{array}\right], \quad V U=\left[\begin{array}{ccc}
v_{1} u_{1} & 0 & v_{1} u_{2} \\
v_{2} u_{1} & 0 & v_{2} u_{2} \\
0 & v_{2} u_{2} & 0
\end{array}\right] .
$$

The one-to-one maps from $\mathcal{A}=\{a, b\}$ to $\mathcal{U V}$ and from $\mathcal{B}=\left\{a^{\prime}, b^{\prime}, c^{\prime}, d^{\prime}\right\}$ to $\mathcal{V U}$ 
are described in the tables below

\begin{tabular}{|l|l|}
\hline$a$ & $u_{1} v_{1}$ \\
\hline$b$ & $u_{2} v_{2}$ \\
\hline
\end{tabular},$\quad$\begin{tabular}{|l|l|}
$a^{\prime}$ & $v_{1} u_{1}$ \\
\hline$b^{\prime}$ & $v_{2} u_{2}$ \\
\hline$c^{\prime}$ & $v_{2} u_{1}$ \\
\hline$d^{\prime}$ & $v_{1} u_{2}$ \\
\hline
\end{tabular}

An elementary strong shift equivalence between $\mathrm{A}=(Q, E)$ and $\mathrm{B}=\left(Q^{\prime}, E^{\prime}\right)$, enables the construction of an irreducible sofic shift $Z$ on the alphabet $\mathcal{U} \cup \mathcal{V}$ as follows. The sofic shift $Z$ is defined by the automaton $\mathrm{C}=\left(Q \cup Q^{\prime}, F\right)$, where the symbolic adjacency matrix $C$ of $\mathrm{C}$ is

$$
\begin{array}{ccc} 
& Q & Q^{\prime} \\
Q & {\left[\begin{array}{cc}
0 & U \\
V & 0
\end{array}\right]}
\end{array}
$$

The shift $Z$ is called the bipartite shift defined by $U, V$ (see Figure 5 ). An edge of $C$ labeled by $\mathcal{U}$ goes from a state in $Q$ to a state in $Q^{\prime}$. An edge of $C$ labeled by $\mathcal{V}$ goes from a state in $Q^{\prime}$ to a state in $Q$. Hence, a path of $\mathrm{C}$ goes from a state in $Q \cup Q^{\prime}$ to a state in $Q \cup Q^{\prime}$, its domain is included either in $Q$ or in $Q^{\prime}$, and its image is included either in $Q$ or in $Q^{\prime}$. If a path of $C$ has domain included in $P$ and the image included in $P^{\prime}$, we say that it has type $\left(P, P^{\prime}\right)$.

Remark that the second higher power of $Z$ is the disjoint union of $X$ and $Y$ since

$$
C^{2}=\left[\begin{array}{cc}
U V & 0 \\
0 & V U
\end{array}\right]
$$

Note also that $\mathrm{C}$ is a right Fischer cover (i.e. is minimal).

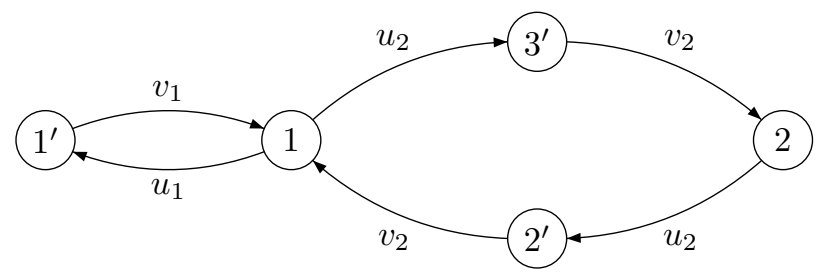

Figure 5: The bipartite shift $Z$ of the shifts $X$ and $Y$ in Figure 4 . The word $u_{1} v_{1}$ has type $(Q, Q)$ and corresponds to the word $a$ in $X$.

\section{A syntactic invariant for conjugacy}

In this section, we prove that the syntactic graph is an invariant for the conjugacy of irreducible sofic shifts. 
TheOREm 4 Let $X$ and $Y$ be two irreducible sofic shifts. If $X$ and $Y$ are conjugate, then their syntactic graphs are isomorphic and the isomorphism preserves the labels.

We give a few lemmas before proving Theorem 4 .

Let $X$ (respectively $Y$ ) be an irreducible sofic shift whose symbolic adjacency matrix of its right Fischer cover is a $(Q \times Q)$-matrix (respectively $\left(Q^{\prime} \times Q^{\prime}\right)$ matrix) denoted by $A$ (respectively by $B$ ). We assume that $A$ and $B$ are elementary strong shift equivalent through a pair of matrices $(U, V)$. The corresponding alphabets are denoted $\mathcal{A}, \mathcal{B}, \mathcal{U}$, and $\mathcal{V}$ as before. We denote by $f$ a one-to-one map from $\mathcal{A}$ to $\mathcal{U V}$ which transforms $A$ into $U V$ and by $g$ a one-to-one map from $\mathcal{B}$ to $\mathcal{V U}$ which transforms $B$ into $V U$. Let $Z$ be the bipartite irreducible sofic shift associated to $U, V$. We denote by $S(X)$ (respectively $S(Y), S(Z)$ ) the syntactic semigroup of $X$ (respectively $Y, Z$ ).

Remark that $w \in S(Z)$ has type $(Q, Q)$ if and only if $w \neq 0$ and $w \in(f(\mathcal{A}))^{*}$, and $w$ has type $\left(Q^{\prime}, Q^{\prime}\right)$ if and only if $w \neq 0$ and $w \in(g(\mathcal{B}))^{*}$.

LEmma 5 Elements of $S(Z)$ in a same non null $\mathcal{H}$-class have the same type.

Proof We show the property for the $(Q, Q)$-type. Let $w \in H$ and $w$ of type $(Q, Q)$. If $w=w^{\prime} v$ with $w^{\prime}, v \in S(Z)$, then $w^{\prime}$ has type $(Q, *)$. If $w=z w^{\prime}$ with $z, w^{\prime} \in S(Z)$, then $w^{\prime}$ has type $(*, Q)$. Thus, $w \mathcal{H} w^{\prime}$ implies that $w^{\prime}$ has type $(Q, Q)$.

The $\mathcal{H}$-classes of $S(Z)$ containing elements of type $(Q, Q)$ (respectively $\left(Q^{\prime}, Q^{\prime}\right)$ ) are called $(Q, Q)$ - $\mathcal{H}$-classes (respectively $\left(Q^{\prime}, Q^{\prime}\right)$ - $\mathcal{H}$-classes).

Let $w=a_{1} \ldots a_{n}$ be an element of $S(X)$, we define the element $f(w)$ as $f\left(a_{1}\right)$ $\ldots f\left(a_{n}\right)$. Note that this definition is consistent since if $a_{1} \ldots a_{n}=a_{1}^{\prime} \ldots a_{m}^{\prime}$ in $S(X)$, then $f\left(a_{1}\right) \ldots f\left(a_{n}\right)=f\left(a_{1}^{\prime}\right) \ldots f\left(a_{m}^{\prime}\right)$ in $S(Z)$. Similarly we define an element $g(w)$ for any element $w$ of $S(Y)$.

Conversely, let $w$ be an element of $S(Z)$ belonging to $f(\mathcal{A})^{*}\left(\subseteq(\mathcal{U V})^{*}\right)$. Then $w=f\left(a_{1}\right) \ldots f\left(a_{n}\right)$, with $a_{i} \in \mathcal{A}$. We define $f^{-1}(w)$ as $a_{1} \ldots a_{n}$. Similarly we define $g^{-1}(w)$. Again these definitions and notations are consistent. Thus $f$ is a semigroup isomorphism from $S(X)$ to the subsemigroup of $S(Z)$ of transition functions defined by the words in $(f(\mathcal{A}))^{*}$. Notice that $f(0)=0$ if $0 \in S(X)$. Analogously, $g$ is a semigroup isomorphism from $S(Y)$ to the subsemigroup of $S(Z)$ of transition functions defined by the words in $(g(\mathcal{B}))^{*}$.

Lemma 6 Let $w, w^{\prime} \in S(Z)$ of type $(Q, Q)$. Then $w \mathcal{H} w^{\prime}$ in $S(Z)$ if and only if $f^{-1}(w) \mathcal{H} f^{-1}\left(w^{\prime}\right)$ in $S(X)$.

Proof Let $w=f\left(a_{1}\right) \ldots f\left(a_{n}\right)$ and $w^{\prime}=f\left(a_{1}^{\prime}\right) \ldots f\left(a_{m}^{\prime}\right)$, with $a_{i}, a_{j}^{\prime} \in \mathcal{A}$. We have $w=w^{\prime} v$ with $v \in S(Z)$ if and only if $v=f\left(\bar{a}_{1}\right) \ldots f\left(\bar{a}_{r}\right)$ with $\bar{a}_{i} \in \mathcal{A}$ and $f\left(a_{1}\right) \ldots f\left(a_{n}\right)=f\left(a_{1}^{\prime}\right) \ldots f\left(a_{m}^{\prime}\right) f\left(\bar{a}_{1}\right) \ldots f\left(\bar{a}_{r}\right)$. This is equivalent to $a_{1} \ldots a_{n}=$ $a_{1}^{\prime} \ldots a_{m}^{\prime} \bar{a}_{1} \ldots \bar{a}_{r}$, that is $f^{-1}(w) S(Z)^{1} \subseteq f^{-1}\left(w^{\prime}\right) S(Z)^{1}$. Analogously, we have $w^{\prime}=w v^{\prime}$ with $v^{\prime} \in S(Z)$, if and only if $f^{-1}\left(w^{\prime}\right) S(Z)^{1} \subseteq f^{-1}(w) S(Z)^{1}$. This proves that $w \mathcal{R} w^{\prime}$ in $S(Z)$ if and only if $f^{-1}(w) \mathcal{R} f^{-1}\left(w^{\prime}\right)$ in $S(X)$. In the same 
way, one can prove the same statement for the relation $\mathcal{L}$ and hence for the relation $\mathcal{H}$.

A similar statement holds for $\left(Q^{\prime}, Q^{\prime}\right)-\mathcal{H}$-classes.

Lemma 7 Let $w, w^{\prime} \in S(Z)$ of type $(Q, Q)$. Then $w \leq \mathcal{J} w^{\prime}$ in $S(Z)$ if and only if $f^{-1}(w) \leq_{\mathcal{J}} f^{-1}\left(w^{\prime}\right)$ in $S(X)$. This implies that $w \mathcal{J} w^{\prime}$ in $S(Z)$ if and only if $f^{-1}(w) \mathcal{J} f^{-1}\left(w^{\prime}\right)$ in $S(X)$.

Proof The first statement can be prooved as in the previous lemma.

Similar results hold between $S(Y)$ and $S(Z)$. As a consequence we get the following lemma.

LEMma 8 The bijection $f$ between $S(X)$ and the elements of $S(Z)$ in $(f(\mathcal{A}))^{*}$, induces a bijection between the non null $\mathcal{H}$-classes of $S(X)$ and the $(Q, Q)-\mathcal{H}$ classes of $S(Z)$. Moreover this bijection keeps the relations $\mathcal{J}, \leq_{\mathcal{J}}$ and the rank of the $\mathcal{H}$-classes.

A similar statement holds for the bijection $g$.

We now come to the main lemma, which shows the link between the elementary strong shift equivalence of the symbolic adjacency matrices and the conjugacy of some idempotents in the semigroup of the bipartite shift. This link is the key point of the invariant.

Lemma 9 Let $H$ be a regular $(Q, Q)-\mathcal{H}$-class of $S(Z)$. Then there is a regular $\left(Q^{\prime}, Q^{\prime}\right)-\mathcal{H}$-class in the same $\mathcal{D}$-class as $H$.

Proof Let $e \in S(Z)$ be an idempotent element of type $(Q, Q)$. Let $u_{1} v_{1} \ldots u_{n} v_{n}$ in $(\mathcal{U V})^{*}$ such that $e=u_{1} v_{1} \ldots u_{n} v_{n}$. We define $\bar{e}=v_{1} \ldots u_{n} v_{n} u_{1}$. Thus $e u_{1}=u_{1} \bar{e}$ in $S(Z)$. Remark that $\bar{e}$ depends on the choice of the word $u_{1} v_{1} \ldots$ $u_{n} v_{n}$ representing $e$ in $S(Z)$.

If $w$ denotes $v_{1} \ldots u_{n} v_{n}$ and $v$ denotes $u_{1}$, we have $e=v w$ and $\bar{e}=w v$. It follows that $e$ and $\bar{e}$ are conjugate, thus $e^{2}=e$ and $\bar{e}^{2}$ are conjugate. Moreover

$$
\bar{e}^{3}=w v w v w v=w e e v=w e v=w v w v=\bar{e}^{2} .
$$

Thus $\bar{e}^{2}$ is an idempotent conjugate to the idempotent $e$. As a consequence $e$ and $\bar{e}^{2}$ belong to a same $\mathcal{D}$-class of $S(Z)$ (see Section 2), and $\bar{e}^{2} \neq 0$. The result follows since $\bar{e}^{2}$ is of type $\left(Q^{\prime}, Q^{\prime}\right)$.

Note that the number of regular $(Q, Q)-\mathcal{H}$-classes and the number of regular $\left(Q^{\prime}, Q^{\prime}\right)$ - $\mathcal{H}$-classes in a same $\mathcal{D}$-class of $S(Z)$, may be different in general.

We now prove Theorem 4 .

Proof[of Theorem 4] By Nasu's Theorem [19] we can assume, without loss of generality, that the symbolic adjacency matrices of the right Fischer covers of 
$X$ and $Y$ are elementary strong shift equivalent. We define the bipartite shift $Z$ as above.

Let $D$ be a non null regular $\mathcal{D}$-class of $S(X)$. Let $H$ be a regular $\mathcal{H}$-class of $S(X)$ contained in $D$. Let $H^{\prime \prime}=f(H)$. By Lemma 8, the groups $H$ and $H^{\prime \prime}$ are isomorphic. Let $D^{\prime \prime}$ the $\mathcal{D}$-class of $S(Z)$ containing $H^{\prime \prime}$. By Lemma 9, there is at least one regular $\left(Q^{\prime}, Q^{\prime}\right)-\mathcal{H}$-class $K^{\prime \prime}$ in $D^{\prime \prime}$, which is isomorphic to $H^{\prime \prime}$. Let $H^{\prime}=g^{-1}\left(K^{\prime \prime}\right)$ and let $D^{\prime}$ be the $\mathcal{D}$-class of $S(Y)$ containing $H^{\prime}$. By Lemma 8 , the groups $H^{\prime}$ and $K^{\prime \prime}$ are isomorphic. Hence the groups $H$ and $H^{\prime}$ are isomorphic.

By Lemmas 8 and 9 , we have that the above construction of $D^{\prime}$ from $D$ is a bijective function $\varphi$ from the non null regular $\mathcal{D}$-classes of $S(X)$ onto the non null regular $\mathcal{D}$-classes of $S(Y)$. Moreover the characteristic group of $D$ is isomorphic to the characteristic group of $\varphi(D)$ and, by Lemma 8, the rank of $D$ is equal to the rank of $\varphi(D)$.

We now consider two non null regular $\mathcal{D}$-classes $D_{1}$ and $D_{2}$ of $S(X)$. By Lemma 8 and Lemma $9, D_{1} \leq_{\mathcal{J}} D_{2}$ if and only if $\varphi\left(D_{1}\right) \leq_{\mathcal{J}} \varphi\left(D_{2}\right)$. It follows that the syntactic graphs of $S(X)$ and $S(Y)$ are isomorphic through the bijection $\varphi$.

\subsection{The reducible case}

Nasu's Classification Theorem holds for reducible sofic shifts by the use of right Krieger covers instead of right Fischer covers [19]. This enables the extension of our result to the case of reducible sofic shifts.

Let $X \subseteq \mathcal{A}^{\mathbb{Z}}$ be a shift. We define

$$
X_{-}=\left\{x_{-} \mid x \in X\right\},
$$

where for $x \in \mathcal{A}^{\mathbb{Z}}$, we denote by $x_{-}$the left infinite word $\ldots x_{-2} x_{-1} x_{0}$. The equivalence relation $\kappa$ on $X_{-}$is defined as follows. Let $x, y \in X_{-}$,

$$
x \kappa y \Leftrightarrow\left\{u \in \mathcal{A}^{+} \mid x u \in X_{-}\right\}=\left\{u \in \mathcal{A}^{+} \mid y u \in X_{-}\right\} .
$$

If $X$ is a sofic shift, the equivalence classes of $\kappa$ are finitely many [15]. The right Krieger cover of $X$ is defined as the automaton labeled by $\mathcal{A}$ in which the states are the $\kappa$-classes $[x]$ with $x \in X_{-}$, and there is a an edge labeled $a$ from $[x]$ to $[x a]$ if $x a \in X_{-}$. The analogous of Theorem 2 for (possibly) reducible sofic shifts is the following.

Theorem 10 [19, Theorem 3.3] Let $X$ and $Y$ be sofic shifts and let $A$ and $B$ be the symbolic adjacency matrices of the right Krieger covers of $X$ and $Y$, respectively. Then $X$ and $Y$ are conjugate if and only if $A$ and $B$ are strong shift equivalent within right Krieger covers.

Hence we can define the syntatic graph of a reducible shift $X$ as the graph of the regular $\mathcal{D}$-classes of the transition semigroup of its right Krieger cover. The result of Theorem 4 is extended as follows for reducible sofic shifts. 
TheOREm 11 Let $X$ and $Y$ be two sofic shifts. If $X$ and $Y$ are conjugate, then their syntactic graphs are isomorphic and the isomorphism preserves the labels.

An effective procedure to construct the right Krieger cover of a sofic shift is described in [19]. First, one constructs the (unique) minimal deterministic automaton with one initial state recognizing the language of finite blocks of the shift. Next, one erases all the states which are not the end of any left-infinite path. This automaton turns out to be the right Krieger cover of the shift. For instance, the right Krieger cover of the even shift in Figure 1, is illustrated in Figure 6 .

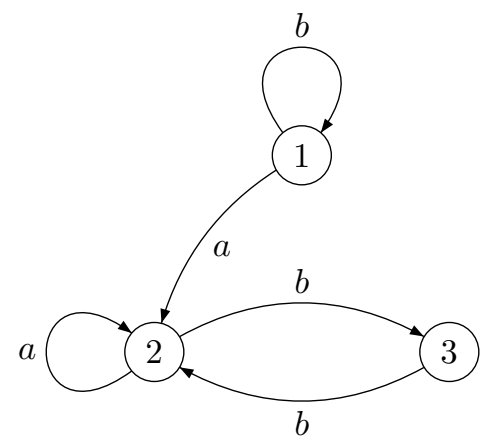

Figure 6: The right Krieger cover of the even shift $X$ described in Figure 1. Notice that, although the shift $X$ is irreducible, the right Fisher cover of $X$ does not coincide with its right Krieger cover.

\section{How dynamic is this invariant?}

In this section, we briefly compare the syntactic invariant with other classical conjugacy invariants. We refer to [18] for their definitions and properties.

First, one can remark that the syntactic invariant does not capture all the dynamics. Two sofic shifts can have the same syntactic graph and a different entropy, as shown in the example of Figure 7.

The comparison with the zeta function is more interesting. Recall that the zeta function of a shift $X$ is $\zeta(X)=\exp \sum_{n>1} p_{n} \frac{z^{n}}{n}$, where $p_{n}$ is the number of bi-infinite words $x \in X$ such that $\sigma^{n}(x)=x$. We give in Figure 8 an example of two irreducible sofic shifts which have the same zeta function and different syntactic graphs.

The following characterization of irreducible shifts of finite type fits naturally in our framework. It is of course well-known and can be obtained for instance from the characterization of syntactic semigroups of local languages (see [9]), and the characterization of syntactic semigroups of irreducible sofic shifts (see [5]), or also from [11]. 

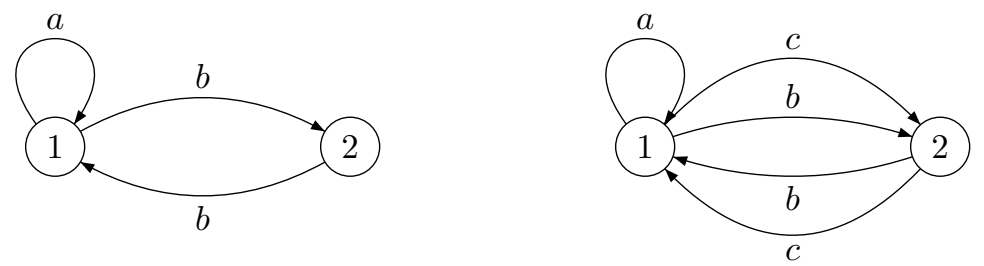

Figure 7: The two above sofic shifts $X, Y$ have the same syntactic graph and different entropies. Indeed, we have $b=c$ in the syntactic semigroup of $Y$. Hence the shifts $X$ and $Y$ have the same syntactic semigroup.
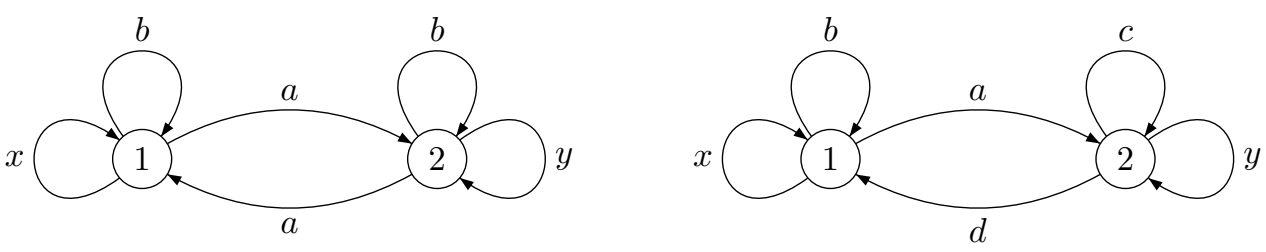

Figure 8: Two sofic shifts $X, Y$ which have the same zeta function $\frac{1}{1-4 z+z^{2}}$ (see for instance [18, Theorem 6.4.8], or [2] for the computation of the zeta function of a sofic shift), and different syntactic graphs. Indeed the syntactic graph of $X$ is $(\operatorname{rank} 2, \mathbb{Z} / 2 \mathbb{Z}) \rightarrow(\operatorname{rank} 1, \mathbb{Z} / \mathbb{Z})$ while the syntactic graph of $Y$ has only one node $($ rank $1, \mathbb{Z} / \mathbb{Z})$. Thus they are not conjugate. Notice that $Y$ is a shift of finite type.

Proposition 12 An irreducible sofic shifts is of finite type if and only its syntactic graph is reduced to one node of rank 1 representing the trivial group.

Proof Let $X$ be an irreducible shift of finite type. It is well known that $X$ is conjugate to an edge shift, that is a sofic shift with a presentation in which the labels of the edges are all different (or, in other words, a finite multigraph which is not labeled). Hence, by Theorem 4 , we can suppose that $X$ is an edge shift. Let $S(X)$ be the syntactic semigroup of $X$. Each non null element of $S(X)$ has rank 1 because it determines an initial state and a terminal state. Moreover it can be easily seen that for $x, y \in S(X) \backslash\{0\}$ we have $x \mathcal{R} y$ if and only if $x$ and $y$ have the same domain, and $x \mathcal{L} y$ if and only if they have the same image. This means that $S(X)$ contains only one $\mathcal{D}$-class of rank 1 and that the $\mathcal{H}$-classes contain exactly one element.

For the converse, suppose that the syntactic graph of an irreducible sofic shift $X$ is reduced to one node of rank 1 representing the trivial group. By [18, Theorem 3.4.17], it suffices to prove that all sufficiently long and non null words in the syntactic semigroup $S(X)$ of $X$ have rank 1. By [20, Chapter 3 Proposition 1.12], we have that all sufficiently long words in $S(X)$ are of the 
form $u v w$, where $v$ is an idempotent of $S(X)$. Being each idempotent of rank 1 , we have that each non null word of the form uvw has rank 1 .

Another interesting class of irreducible sofic shifts can be characterized with the syntactic invariant. It is the class of aperiodic sofic shifts [1].

Let $x \in X$, we denote by period $(x)$ the least positive integer $n$ such that $\sigma^{n}(x)=x$ if such an integer exists. It is equal to $\infty$ otherwise.

Let $X, Y$ be two shifts and let $\phi: X \rightarrow Y$ be a block map. The map is said aperiodic if period $(x)=\operatorname{period}(\phi(x))$ for any $x \in X$ with finite period. Roughly speaking, such a factor map $\phi$ does not make periods decrease.

A sofic shift is aperiodic if it is the image of a shift of finite type under an aperiodic block map. An aperiodic presentation is a presentation in which for every $u \in \mathcal{A}^{+}$, whenever there is a cycling path labeled $u^{n}$

$$
p_{1} \stackrel{u}{\rightarrow} p_{2} \stackrel{u}{\rightarrow} \ldots \stackrel{u}{\rightarrow} p_{n} \stackrel{u}{\rightarrow} p_{1},
$$

one has $p_{i}=p_{1}$ for each $i=2, \ldots, n$.

Proposition 13 A sofic shift is aperiodic if and only if it has an aperiodic presentation.

Proof Let $X$ be an aperiodic sofic shift. Hence $X=\phi(Y)$, where $\phi$ is an aperiodic block map and $Y$ a shift of finite type. Notice that we can always suppose that $Y$ is an edge shift and that $\phi$ has no memory nor anticipation. Hence a presentation $\mathrm{A}$ of $X$ is given by the not labeled presentation $\mathrm{G}$ of $Y$ in which the label of an edge $e$ is the letter $\phi(e)$ (we identify the block map $\phi$ with the local rule $\delta$ defining it). Moreover, we can always suppose that there is at most one edge from a given state $p$ to a given state $q$ in $\mathrm{G}$. We have that the presentation A is aperiodic. Indeed, let

$$
p_{1} \stackrel{u}{\rightarrow} p_{2} \stackrel{u}{\rightarrow} \ldots \stackrel{u}{\rightarrow} p_{n} \stackrel{u}{\rightarrow} p_{1}
$$

be a path in A. Notice that we can always suppose that the configuration $u^{\infty} \in$ $X$, obtained by repeating infinitely many times the word $u$ in both directions, has period $h=|u|$. Moreover, in $\mathrm{G}$ there is a path $v$

$$
p_{1} \stackrel{e_{1}^{(1)} \ldots e_{h}^{(1)}}{\longrightarrow} p_{2} \stackrel{e_{1}^{(2)} \ldots e_{h}^{(2)}}{\longrightarrow} \ldots \stackrel{e_{1}^{(n-1)} \ldots e_{h}^{(n-1)}}{\longrightarrow} p_{n} \stackrel{e_{1}^{(n)} \ldots e_{h}^{(n)}}{\longrightarrow} p_{1},
$$

such that $\phi\left(e_{1}^{(i)} \ldots e_{h}^{(i)}\right)=u$, for each $i=1, \ldots, n$. Being $\phi\left(v^{\infty}\right)=u^{\infty}$, the configuration $v^{\infty} \in Y$ must have period $h$. This implies $e_{1}^{(i)} \ldots e_{h}^{(i)}=e_{1}^{(1)} \ldots e_{h}^{(1)}$ for each $i=2, \ldots, n$. In particular we have $p_{i}=p_{1}$ for each $i=2, \ldots, n$.

For the converse, suppose that $X$ is a sofic shift with an aperiodic presentation A. Let $Y$ be the edge shift whose presentation is the underlying graph $\mathrm{G}$ of A. Let $\phi: Y \longrightarrow X$ be the labeling map. We have that $\phi$ is aperiodic. Indeed let $x$ be a configuration of $Y$ with period $n$. Hence in $\mathrm{G}$ there is the path

$$
p_{1} \stackrel{e_{1}}{\longrightarrow} p_{2} \stackrel{e_{2}}{\longrightarrow} \ldots \stackrel{e_{n-1}}{\longrightarrow} p_{n} \stackrel{e_{n}}{\longrightarrow} p_{1},
$$


where $x=\left(e_{1} e_{2} \ldots e_{n}\right)^{\infty}$. Hence $\phi(x)=\left(a_{1} a_{2} \ldots a_{n}\right)^{\infty}$, where $a_{i}$ is the label of the edge $e_{i}$. Suppose that $a_{1} \ldots a_{n}=\left(a_{1} \ldots a_{h}\right)^{k}$, with $h k=n$. Hence in A we have the path

$$
p_{i} \stackrel{a_{i} \ldots a_{h} a_{1} \ldots a_{i-1}}{\longrightarrow} p_{h+i} \stackrel{a_{i} \ldots a_{h} a_{1} \ldots a_{i-1}}{\longrightarrow} \ldots p_{(k-1) h+i} \stackrel{a_{i} \ldots a_{h} a_{1} \ldots a_{i-1}}{\longrightarrow} p_{i},
$$

for each $i=1, \ldots, h$. Being the presentation of $X$ aperiodic, one has $p_{i}=$ $p_{h+i}=\cdots=p_{(k-1) h+i}$ for each $i=1, \ldots, h$. This means that the edges

$$
\begin{gathered}
p_{i} \stackrel{e_{i}}{\longrightarrow} p_{i+1} \\
p_{h+i} \stackrel{e_{h+i}}{\longrightarrow} p_{h+i+1} \\
\vdots \\
p_{(k-1) h+i} \stackrel{e_{(k-1) h+i}}{\longrightarrow} p_{(k-1) h+i+1},
\end{gathered}
$$

have same initial state, same final state and same label (where the state $p_{n+1}$ is defined as $p_{1}$ ). Thus they coincide and this implies $h=n$ and $k=1$. Hence $\operatorname{period}(\phi(x))=n$.

Proposition 14 The right Fischer cover of an irreducible aperiodic sofic shift is an aperiodic presentation.

Proof Let $A$ be an aperiodic presentation of a sofic shift $X$. Let us assume that $A$ is not deterministic. We compute from $A$ a deterministic presentation $B$ by the well known subset construction (see for instance [18, Section 3.3]). We show that $B$ is an aperiodic presentation.

Suppose that in B there is a cycling path labeled by $u^{n}$

$$
P_{1} \stackrel{u}{\rightarrow} P_{2} \stackrel{u}{\rightarrow} \ldots \stackrel{u}{\rightarrow} P_{n} \stackrel{u}{\rightarrow} P_{1},
$$

where $u$ is a word and each $P_{i}$ is a state of B identified with a subset of the states of A. Let $P$ and $Q$ be two subsets of the states of A. Recall that in B there is a unique path from $P$ to $Q$ labeled $u$, if and only if $Q$ is the set of all states $q$ in A for which there is at least one state $p$ in $P$ and a path in A from $p$ to $q$ labeled $u$. If such a path exists, the state $Q$ is denoted by $P \cdot u$. It follows that, for each state $p_{j} \in P_{1}$, there is a left infinite path $\left(q_{j, 1-(i+1)} \stackrel{u}{\rightarrow} q_{j, 1-i}\right)_{i \geq 0}$ labeled by ${ }^{\omega} u$ (that is the left infinite word obtained by repeating infinitely many times the word $u$ on the left), where $q_{j, 1}=p_{j}$ and $q_{j, i} \in P_{i \bmod n}$ for each $i \geq 0$. Since the number of states is finite, there are two positive integer $m$ and $l$ and a finite path in A such that $q_{j, 1-(m+l)} \stackrel{u^{l}}{\longrightarrow} q_{j, 1-m} \stackrel{u^{m}}{\longrightarrow} q_{j, 1}=p_{j}$ with $q_{j, 1-(m+l)}=q_{j, 1-m}$. Since $A$ is aperiodic, one can set $l=1$. Moreover, one can always suppose that $m$ does not depend on $j$. Let $k=(1-m) \bmod n$. We denote by $Q_{k}$ the set of 
all states $q_{j, 1-m}$. Thus $Q_{k} \cdot u^{m}=P_{1}$. Moreover, we have

$$
\begin{aligned}
Q_{k} & \subseteq Q_{k} \cdot u \subseteq Q_{k} \cdot u^{2} \cdots \subseteq Q_{k} \cdot u^{m}=P_{1} \\
& \subseteq Q_{k} \cdot u^{m+1}=P_{2} \\
& \subseteq Q_{k} \cdot u^{m+2}=P_{3} \\
& \cdots \\
& \subseteq Q_{k} \cdot u^{m+n+1}=P_{1} .
\end{aligned}
$$

It follows that $P_{1} \subseteq P_{2} \subseteq P_{3} \cdots \subseteq P_{n} \subseteq P_{1}$, and finally $P_{1}=P_{2}=\cdots=P_{n}$.

The right Fischer cover of the shift is obtained by state merging of states of B having the same future. Thus, if B is an aperiodic presentation, its right Fischer cover also. It is known that the right Fischer cover of an irreducible shift has a strongly connected graph.

A characterization of irreducible aperiodic sofic shifts is the following.

PROPOSITION 15 An irreducible sofic shift is aperiodic if and only if its syntactic graph contains only trivial groups.

Proof Let $X$ be an irreducible aperiodic sofic shift and let $S(X)$ be the syntactic semigroup of $X$. If $e$ is an idempotent of $S(X)$ and $u \in S(X)$ is such that $u \mathcal{H} e$, there exists $n \geq 1$ such that $u^{n}=e$. Being the right Fischer cover of $X$ aperiodic, the function $u$ coincides with $e$ at each state $p$ such that $e(p)=p$. If $e(p) \neq p$ and $u(p)=q$, we have that $q$ is in the image of $e$ because this latter coincides with the image of $u$. Hence $e(q)=q$ and then $u(q)=q$. This implies $e(p)=u^{n}(p)=u^{n-1}(q)=q=u(p)$. Thus $u=e$. Hence all the regular $\mathcal{H}$-classes of $S(X)$ are trivial.

For the converse, suppose that the syntactic graph of an irreducible sofic shift $X$ has only trivial groups. Let $u^{n}$ be the label of a cycle

$$
p_{1} \stackrel{u}{\rightarrow} p_{2} \stackrel{u}{\rightarrow} \ldots \stackrel{u}{\rightarrow} p_{n} \stackrel{u}{\rightarrow} p_{1} .
$$

Without loss of generality, we can assume that $u^{n}$ is idempotent (indeed there is always a power of $u^{n}$ which is idempotent). Being $u^{n+1}, \ldots, u^{2 n-1}$ in the same $\mathcal{H}$-class of $u^{n}$, they must coincide. From $u^{n+i}=u^{n}$ we deduce $p_{i+1}=p_{1}$ for each $i=1, \ldots, n-1$.

Schützenberger's characterization of aperiodic languages (see for instance [20, Chapter 4 Theorem 2.1]) asserts that the set of blocks of an aperiodic sofic shift is a regular star free language.

\section{An invariant for shift equivalence}

We now prove that our invariant for strong shift equivalence is also an invariant of shift equivalence. Although shift equivalence is decidable, even for sofic 
shifts [12], the algorithm is quite intricate. Hence invariants for shift equivalence of sofic shifts, which is equivalent to eventual conjugacy, may be useful. Most known conjugacy invariants are also invariants for shift equivalence.

Two symbolic adjacency matrices $A$ and $B$ with entries in $\mathcal{A}$ and $\mathcal{B}$ respectively, are shift equivalent with lag $l$, where $l$ is a positive integer, if there is a pair of symbolic adjacency matrices $(U, V)$ with entries in disjoint alphabets $\mathcal{U}$ and $\mathcal{V}$ respectively, such that (see [8])

$$
\begin{aligned}
A^{l} \leftrightarrow U V, & B^{l} \leftrightarrow V U, \\
A U \leftrightarrow U B, & V A \leftrightarrow B V .
\end{aligned}
$$

Two matrices are shift equivalent if there is a positive integer $l$ such that they are shift equivalent with lag $l$. Strong shift equivalence implies shift equivalence but the converse is false [13].

In the following theorem we prove that our invariant is also invariant under shift equivalence.

THEOREM 16 Let $X$ and $Y$ be two sofic shifts. If $X$ and $Y$ are shift equivalent, then their syntactic graphs are isomorphic and the isomorphism preserves the labels.

Proof Let $A$ (respectively $B$ ), be the symbolic adjacency matrix of the right Fischer cover of $X$ (respectively of $Y$ ) if $X$ and $Y$ are irreducible, or of the right Krieger cover of $X$ (respectively of $Y$ ) if $X$ and $Y$ are reducible. Suppose that $A$ and $B$ are shift equivalent with lag $\bar{l}$. Notice that $A$ and $B$ are shift equivalent with lag $l$ for each $l \geq \bar{l}$. Moreover, being $A^{l}$ elementary strong shift equivalent to $B^{l}$, they have the same syntactic graph by Theorem 4 .

Hence it suffices to prove that for each symbolic adjacency matrices $A$ and $B$, there is a big enough integer $l$, such that $A$ and $A^{l}$ have the same syntactic graph, and $B$ and $B^{l}$ have the same syntactic graph.

Let $S(X)$ be the syntactic semigroup of $X$. For each idempotent $e \in S(X)$, let $w_{e} \in \mathcal{A}^{*}$ be a word representing $e$. For each $x \in S(X)$ such that $x \mathcal{H} e$, there is a positive integer $h_{x, e}$ such that $u^{h_{x, e}}=e($ recall that a regular $\mathcal{H}$-class is a finite group). We do the same for each idempotent $e^{\prime} \in S(Y)$ and each $y \in S(Y)$ such that $y \mathcal{H} e^{\prime}$ in $S(Y)$. Let

$$
h_{X}=\prod_{\substack{e \in S(X) \\ e^{2}=e}}\left|w_{e}\right| \times \prod_{\substack{x, e \in S(X) \\ e^{2}=e, x \mathcal{H} e}} h_{x, e} .
$$

Let $h=h_{X} \times h_{Y} \times \bar{l}$ and $l=h+1$. Note that $l \geq \bar{l}$.

We prove that $A$ and $A^{l}$ have the same syntactic graph. The same proof holds for $B$ and $B^{l}$. Let $S\left(A^{l}\right)$ be the syntactic semigroup of $A^{l}$. First, notice that the words representing elements of $S\left(A^{l}\right)$ are words labelled in $\mathcal{A}^{l}$. Thus $S\left(A^{l}\right)$ is a isomorphic to a subsemigroup of $S(X)$ and a Green's relation in $S\left(A^{l}\right)$ is still a Green's relation in $S(X)$.

Let $e$ be an idempotent of $S(X)$ and let $D$ be its regular $\mathcal{D}$-class in $S(X)$. Since $e^{l}=e$, the idempotent $e$ is also an idempotent of $S\left(A^{l}\right)$, and the regular 
$\mathcal{D}$-class of $S\left(A^{l}\right)$ containing $e$ is contained in $D$. Moreover, if two idempotents $e$ and $\bar{e}$ are contained in the same $\mathcal{D}$-class of $S(X)$, then they are also contained in the same $\mathcal{D}$-class of $S\left(A^{l}\right)$. Indeed let $x, y \in S(X)$ such that $\bar{e}=x e y$. Let $u$ (resp. $v$ ) a word in $\mathcal{A}^{*}$ representing $x$ (resp. $y$ ). We have, since $e$ and $\bar{e}$ are idempotents,

$$
\bar{e}=u w_{e}^{\left(|u|+\left|w_{e}\right|\right) \frac{h}{\left|w_{e}\right|}+1} e v w_{\bar{e}}^{\left(|v|+\left|w_{\bar{e}}\right|\right) \frac{h}{\left|w_{\bar{e}}\right|}+1}
$$

and

$$
\left|u w_{e}^{\left(|u|+\left|w_{e}\right|\right) \frac{h}{w_{e}}+1}\right|=|u|+\left(|u|+\left|w_{e}\right|\right) h+\left|w_{e}\right|=\left(|u|+\left|w_{e}\right|\right) l .
$$

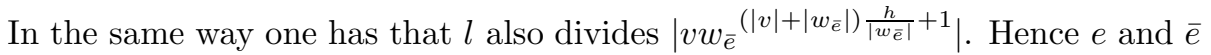
are in the same $\mathcal{D}$-class of $S\left(A^{l}\right)$.

Hence to each regular $\mathcal{D}$-class in $S(X)$ corresponds exactly one regular $\mathcal{D}$ class in $S\left(A^{l}\right)$ and the partial order relation $\leq_{\mathcal{J}}$ is kept.

It remains to prove that for each idempotent $e$, the regular $\mathcal{H}$-classes $H \subseteq$ $S(X)$ and $\bar{H} \subseteq S\left(A^{l}\right)$ containing $e$, coincide. Clearly $\bar{H} \subseteq H$. For the converse, if $x \in H$, we have that $x^{h}=e$ (recall that $h_{x, e}$ divides $\left.h\right)$, and hence $x^{h+1}=$ $x^{l}=x$. Since $x^{l} \in S\left(A^{l}\right)$, we have that $x \in \bar{H}$.

\section{References}

[1] M.-P. BÉAL, Codage Symbolique, Masson, 1993.

[2] M.-P. BÉAL, Puissance extérieure d'un automate déterministe, application au calcul de la fonction zêta d'un système sofique, RAIRO Inform. Théor. Appl., 29 (1995), pp. 85-103.

[3] M.-P. BÉAl, F. Fiorenzi, And F. Mignosi, Minimal forbidden patterns of multi-dimensional shifts. To appear in Internat. J. Algebra Comput., 2004.

[4] M.-P. BÉAL, F. FiOREnZI, AND D. PERrin, The syntactic graph of a sofic shift, in STACS 2004, vol. 2996 of Lecture Notes in Comput. Sci., Springer, Berlin, 2004, pp. 282-293.

[5] D. Beauquier, Minimal automaton for a factorial transitive rational language, Theoret. Comput. Sci., 67 (1989), pp. 65-73.

[6] J. Berstel And D. Perrin, Theory of Codes, Academic Press, New York, 1985.

[7] M. BoyLE, Algebraic aspects of symbolic dynamics, in Topics in symbolic dynamics and applications (Temuco 97), vol. 279 of London Math. Soc. Lecture Notes Ser., Cambridge University Press, Cambridge, 2000, pp. 5788. 
[8] M. Boyle And W. Krieger, Almost markov and shift equivalent sofic systems, in Dynamical Systems, vol. 1342 of Lecture Notes in Mathematics, Springer, 1988, pp. 33-93.

[9] A. De Luca And A. Restivo, A characterization of strictly locally testable languages and its application to subsemigroups of a free semigroup, Inform. and Control, 44 (1980), pp. 300-319.

[10] G. A. HedLund, Endomorphisms and automorphisms of the shift dynamical system, Math. Systems Theory, 3 (1969), pp. 320-337.

[11] N. Jonoska, A conjugacy invariant for reducible sofic shifts and its semigroup characterizations, Israel J. Math., 106 (1998), pp. 221-249.

[12] K. H. Kim And F. W. Roush, An algorithm for sofic shift equivalence, Ergodic Theory Dynam. Systems, 10 (1990), pp. 381-393.

[13] —, Williams's conjecture is false for reducible subshifts, J. Amer. Math. Soc., 5 (1992), pp. 213-215.

[14] B. P. Kitchens, Symbolic Dynamics: one-sided, two-sided and countable state Markov shifts, Springer-Verlag, 1997.

[15] W. Krieger, On sofic systems. I, Israel J. Math., 48 (1984), pp. 305-330.

[16] —, On sofic systems. II, Israel J. Math., 60 (1987), pp. 167-176.

[17] — On a syntactically defined invariant of symbolic dynamics, Ergodic Theory Dynam. Systems, 20 (2000), pp. 501-516.

[18] D. A. Lind And B. H. Marcus, An Introduction to Symbolic Dynamics and Coding, Cambridge, 1995.

[19] M. NAsu, Topological conjugacy for sofic systems, Ergodic Theory Dynam. Systems, 6 (1986), pp. 265-280.

[20] J.-E. Pin, Varieties of formal languages, Foundations of Computer Science, Plenum Publishing Corp., New York, 1986.

[21] K. Thomsen, On the structure of a sofic shift space, Trans. Amer. Math. Soc., 356 (2004), pp. 3557-3619 (electronic).

[22] B. Weiss, Subshifts of finite type and sofic systems, Monats. für Math., 77 (1973), pp. 462-474. 